\title{
Applications and grants of National Natural Scientific Foundation of China's General Program in abnormalities and diseases of locomotor system: a ten-year review
}

\section{Dou Dou}

Department of Health Sciences, National Natural Science Foundation of China, Beijing, China

Correspondence to: Dou Dou, PhD. Department of Health Sciences, National Natural Science Foundation of China, 83 Shuangqing Road, Beijing, China. Email: doudou@nsfc.gov.cn.

\begin{abstract}
Translational medicine is a branch of medical research that attempts to establish a more direct link between basic research and clinical practice. The improvement of clinical management cannot be separated from the progress and breakthrough made in basic biomedical research. As one of the main funding agencies for basic research in China, National Natural Scientific Foundation of China (NSFC) plays an active role in promotion of the development of basic medical research and translational medicine. General Program mainly supports scientists to carry out basic research on bottom-up based topics within the funding scope of NSFC to conduct innovative research and promote a balanced, coordinated and sustained development of all disciplines. Musculoskeletal injury and disease is a major threat to human health worldwide and has become the major cause of disability in China. In this review, we aim to further promote the clinical transformation by summarizing the trends and hotspots of basic research in this field based on the analysis of the data of General Program in abnormalities and diseases of locomotor system over the last decade, including analyses of the number of applications received and projects funded, the distribution of the keywords in applications and grants, distribution of applications and grants of General Program in the secondary application code of $\mathrm{H} 06$ and in the host institution.
\end{abstract}

Keywords: National Natural Scientific Foundation of China (NSFC); general program; funding rate; locomotor system

Submitted Apr 07, 2020. Accepted for publication Jul 03, 2020.

doi: $10.21037 /$ atm-20-3176

View this article at: http://dx.doi.org/10.21037/atm-20-3176

Translational medicine, first appearing as a new term in the paper of The Lancet in 1996 (1), is a branch of medical research that attempts to establish a more direct link between basic research and clinical practice. Agencies that fund basic medical research in many countries play an active role in promotion of the development of basic medical research and translational medicine. Turning discovery into health has been explicitly proposed in National Institute of Health (NIH)-wide strategic plan from 2016 to 2020. The partial aim of Medical Research Council (MRC) strategic plan from 2014 to 2019 was to set priorities of those research which mostly deliver improved health outcomes and bring the benefits to all sections of society. The strategic plan of Canadian Institute of Health Research (CIHR) from 2014-15 to 2018-19 mentioned the promotion of excellence, creativity and breadth in health science and knowledge translation. National Natural Scientific Foundation of China (NSFC) is one of the main funding agencies for basic research in China. The latest reform initiatives of NSFC identified four major funding categories based on scientific natures of research, funding creative and timely ideas to achieve excellence in science, focusing on the frontiers of science in unique ways to lead the cutting edges, supporting application-driven basic research to enable breakthroughs, 


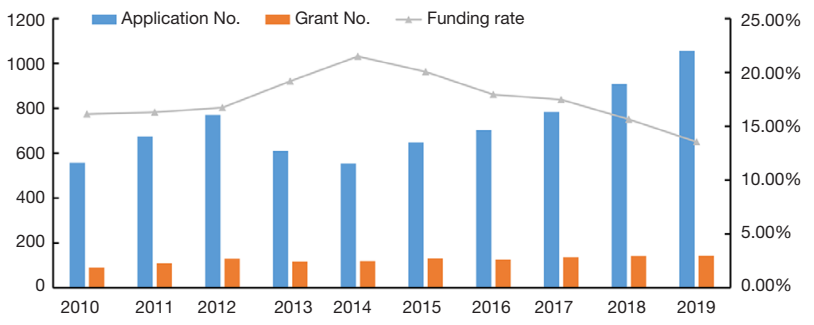

Figure 1 The number of Applications, grants and funding rate for General Program of NSFC in abnormalities and diseases of locomotor system over the past decade.

and encouraging transdisciplinary leading-edge research to promote convergence, which imply to address the core scientific problems and to facilitate the transformation of basic research results (2).

Musculoskeletal injury and disease are a major threat to human health worldwide and has become the major cause of disability in China in 2017 (3). So far, surgery has been the main treatment of it. However, it is not a panacea, and many important problems cannot be solved by surgical treatment. For example, congenital scoliosis is a deformity that occurs immediately after birth, which makes it hard for the patients to receive the best early treatment. Qiu's team found that $11 \%$ of patients with congenital scoliosis in the Han population had rare invalid mutations and a composite inheritance of TBX6 sub-allele (4). Based on the above study, TBX6 gene mutation detection kit for congenital scoliosis has been developed, which helps to reduce the morbidity of congenital scoliosis through early diagnosis. Therefore, the improvement of clinical management cannot be separated from the progress and breakthrough made in basic biomedical research.

General Program is one of the main program types funded by NSFC, the numbers of applications and grants of which are the largest among all types of programs each year. General Program mainly supports scientists to carry out basic research on bottom-up based topics within the funding scope of NSFC to conduct innovative research and promote a balanced, coordinated and sustained development of all disciplines (5). As a result of the bottom-up research, the distribution of General Program in research directions can reflect the concerns and interests of researchers to some extent. When the Department of Health Sciences of NSFC was established in 2009, there are 31 primary codes ( $\mathrm{H} 01$ to $\mathrm{H} 31)$ and many relevant secondary codes of the application code system. The projects applied and funded in the field of musculoskeletal research were mainly distributed in the primary application code of H06 (abnormalities and diseases of locomotor system) and its 13 secondary codes (6). In this review, we aim to further promote the clinical transformation by summarizing the trends and hotspots of basic research in this field based on the analysis of the data of General Program in abnormalities and diseases of locomotor system (excluding tumor) over the last decade.

\section{Applications and grants of General Program in abnormalities and diseases of locomotor system over the past decade}

From 2010 to 2019, 7,266 proposals applying for General Program in abnormalities and diseases of locomotor system were received by the Department of Health Sciences of NSFC, and 1243 projects were funded with a total funding of 7.1254 billion RMB (funding has been divided into direct and indirect cost since 2015, the funding of the projects funded since 2015 in this paper were all direct cost). The number of applications for General Program received by the Department of Health Sciences of NSFC increased rapidly from 558 in 2010 to 1,056 in 2019 . Due to the rapid growth of the number of applications applying for General Program, NSFC developed some new policies to limit application in 2013. For example, applicants could not apply if they received funding for General Program as the principle investigator in the previous year or have not been funded for the General Program for two consecutive years $(7,8)$. Therefore, the numbers of applications for General Program in this field in 2013 and 2014 were decreased compared with that in 2012. Due to the development of the scientific research talents and teams in this field, the number of applications has steadily increased again from 2015 to 2019 (Figure 1). The overall trends of the funded grants were the same as that of applications. The number 
Table 1 Primary and secondary application codes

\begin{tabular}{|c|c|}
\hline Code & Description \\
\hline H0601 & Structural, functional and developmental abnormalities in locomotor system \\
\hline H0602 & Hereditary diseases in locomotor system \\
\hline H0603 & Immune related diseases in locomotor system \\
\hline H0605 & Damage and repair of bone, joint and soft tissue \\
\hline H0606 & Transplantation and reconstruction of bone, joint and soft tissue \\
\hline H0607 & Infection of bone, joint and soft tissue \\
\hline H0608 & Fatigue and recuperation of bone, joint and soft tissue \\
\hline H0611 & Deformity and correction in locomotor system \\
\hline H0612 & Novel technologies for the diagnosis and treatment of diseases in locomotor system \\
\hline H0613 & Other scientific issues of diseases in locomotor system \\
\hline
\end{tabular}

of funded grants increased from 90 in 2010 to 129 in 2012, then dropped to 117 in 2013, and then increased gradually to 143 in 2019 (Figure 1). In the past decade, China's investment in basic research increased year by year, accordingly, the funding rate of General Program in abnormalities and diseases of locomotor system by NSFC also increased from $16.13 \%$ in 2010 to $21.48 \%$ in 2014. However, due to the rapid increase in the number of application, the funding rate has been declined continuously from $21.48 \%$ in 2014 to $13.54 \%$ in 2019 (Figure 1).

\section{Distribution of applications and grants of General Program in the secondary application code of H06 over the past decade}

The primary application codes of Department of Health Sciences are mainly arranged according to the order of organs or systems, and the secondary application codes are arranged in the order from basic to clinical research as well as from structural, functional and developmental abnormalities to diseases, covering both basic and clinical research related to the given organs or systems. Primary application code H06 and its 13 secondary application codes are listed in Table $1(6,9)$.
From 2010 to 2019, the applications and funded grants for General Program were concentrated in the research fields of damage and repair of bone, joint and soft tissue (H0605), and the degenerative diseases of bone, joint and soft tissue (H0609). In contrast, the number of applications and funded grants in field of fatigue and recuperation of bone, joint and soft tissue (H0608) was very small (Figure $2 A$ ), which is consistent with the clinical spectrum of diseases in locomotor system. The funding rate in the research directions on structural, functional and developmental abnormalities and hereditary diseases of locomotor system (H0601 and H0602) has been high for ten years, and the 10-year average funding rate for H0601 and H0602 were $29.05 \%$ and $22.93 \%$ respectively (Figure 2). It was partly because of the better scientific research foundation of researchers in this field, and partly because of the special support given by the Department of Health Sciences to research projects aimed at studying the development and prevention of human rare diseases since 2017. Although the funding rates of H0607 (infection of bone, joint and soft tissue) fluctuated significantly over the past decade, the 10 -year average funding rate of which was 19.77\%, ranking third in General Program (Figure 2). 
A

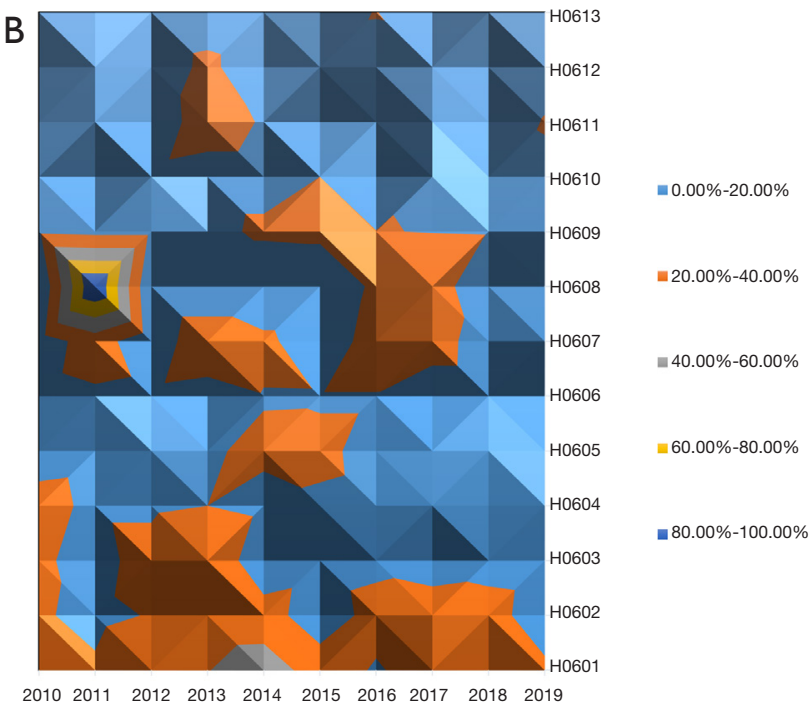

Figure 2 The number of applications, grants and funding rate for General Program of NSFC in the secondary application code of H06 over the past decade (A); the change of funding rate of the secondary application code of $\mathrm{H} 06$ in the past ten years (B).

\section{Keywords in applications and grants of General Program in abnormalities and diseases of locomotor system over the past decade}

With the development of scientific research and the expansion of interdisciplinary cooperation in recent years, the number of keywords in applications for General Program in abnormalities and diseases of locomotor system was also increasing, which has exceeded 2,600 in 2019. The top 10 most frequently used keywords in the applications over the past ten years were stem cells, osteoarthritis, intervertebral disc degeneration, RNA (including microRNA, lncRNA, circRNA), osteoporosis, fracture, tissue engineering, biomechanics, bone defect and osteonecrosis of femoral head, which reflected that the researchers in the field of abnormalities and diseases of locomotor system were more interested in the scientific issues of musculoskeletal degenerative diseases and damage, and in the new theories, methods and techniques such as stem cells, RNA, tissue engineering, etc. (Figure $3 A, B)$. The top 10 keywords in the funded grants were intervertebral disc degeneration, RNA, osteoarthritis, osteoporosis, tissue engineering, fracture, bone defect, osteonecrosis of femoral head and spinal cord injury, nine of which are overlapped with that in applications but in different order, indicating the difference of research level in the scientific teams in these research areas (Figure $3 C, D$ ). Although "spinal cord injury" was not present in the top 10 keywords in the applications, it appears in that of the funded grants, suggesting that the overall level of scientific research in this field might be better (Figure 3C,D)

During the last ten years, a number of new fields have emerged in medicine and related disciplines, such as intestinal flora, pyroptosis, exosome, 3D printing, big data and artificial intelligence. As shown in Figure 4, the applications of these keywords for General Program have grown out of nothing and increased year by year in the field of abnormalities and diseases of locomotor system over the past ten years (Figure $4 A, B$ ). Moreover, some proposals including these keywords, except pyroptosis and big data, have been funded (Figure 4C,D), which suggests that these emerging fields are gradually being integrated into the field of abnormalities and diseases in locomotor system.

\section{Distribution of applications and grants of General Program in the host institution over the past decade}

During the period from 2010 to 2019, all of 7,266 applications for General Program in the field of abnormalities and diseases in locomotor system have been proposed by 291 host institutions in China, among which 1,243 proposals from 106 host institutions were funded. $69.98 \%$ of applications and $82.46 \%$ of grants were concentrated in the top 30 host institutions. In the past ten years, Shanghai Jiao Tong University has ranked first 
A

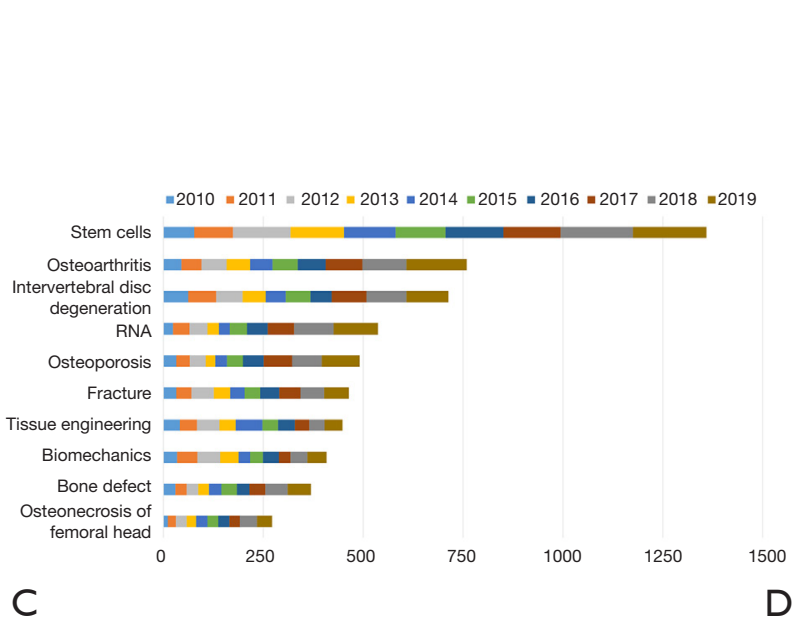

B

D

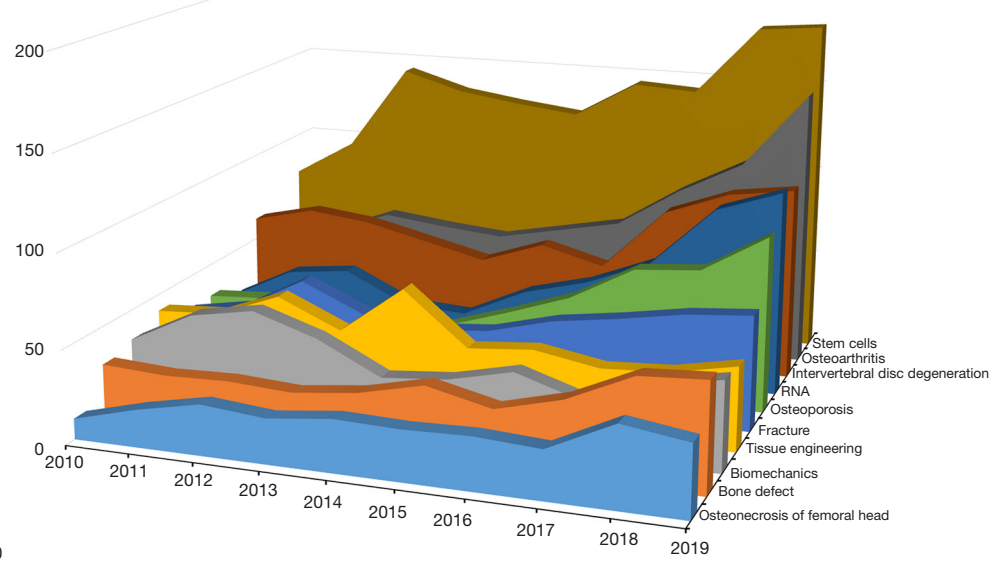

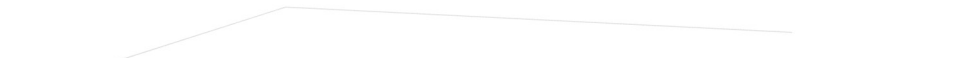

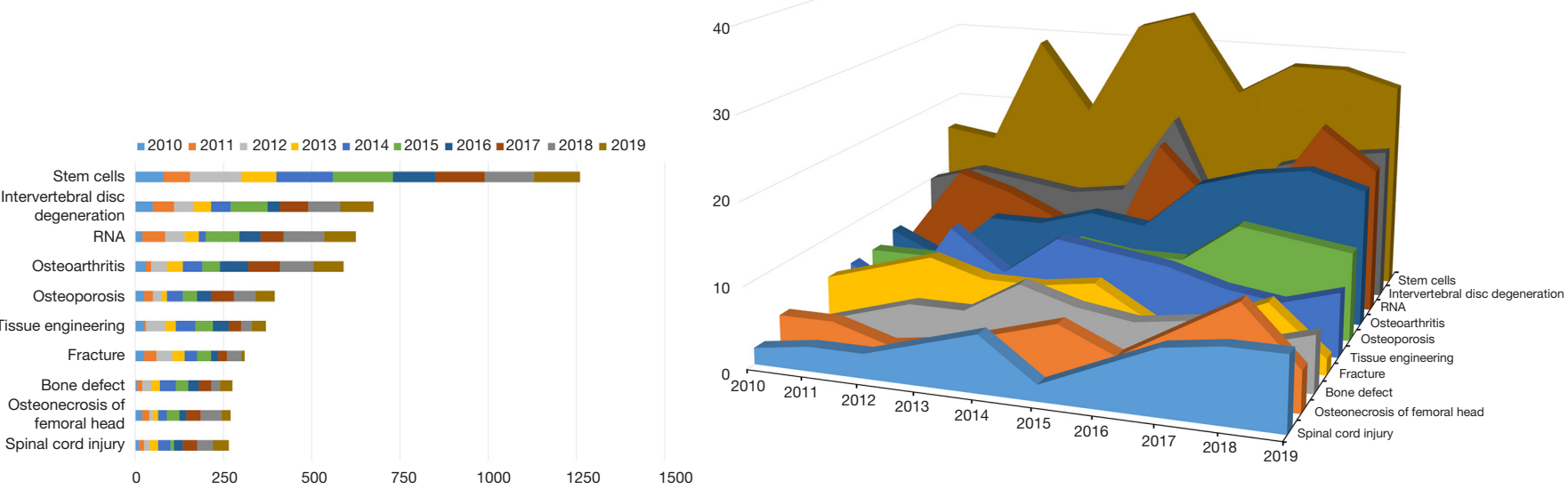

Figure 3 Top 10 keywords in applications (A) and grants (C), and the number of projects involved in the top 10 keywords in applications (B) and grants (D) for General Program in abnormalities and diseases of locomotor system over the past 10 years.

in the number of both applications and grants, accounting for $8.43 \%$ and $10.62 \%$ respectively. There were 32 host institutions, each of which has been funded for only one project over the past ten years. Although the numbers of applications from Tianjin Medical University and Peking Union Medical Hospital were less than that of other top 30 institutions, the funding rates of them were $42.37 \%$ and $36.71 \%$, taking the top 2 places (Figure $5 A$ ).

The funded projects in different host institutions were unevenly distributed among the 13 secondary codes of H06. Shanghai Jiao Tong University has the largest number of projects funded in most of the secondary codes including H0602, H0604, H0605, H0606, H0607 and H0609, Sun Yat-Sen University in the secondary code of H0603 and H0608, Peking Union Medical College Hospital in the secondary code of H0601 and H0611, and Fudan University in the secondary code of H0610 (Figure 5B). This indicated that these host institutions might have the research advantages or high-level research teams in the relevant research directions.

\section{Future and direction}

The scope of H06 mainly supports the research on the abnormalities in structure, function and development of the bone, joint, muscle and ligament, and the research in the etiology, pathogenesis, diagnosis, prevention and treatment of the musculoskeletal diseases including the genetic diseases, the immune-related diseases, inflammation and infection, injury and repair, grafting and reconstruction, fatigue and recuperation, degenerative disorders, sports injury, deformity and correction, and non-neoplastic 

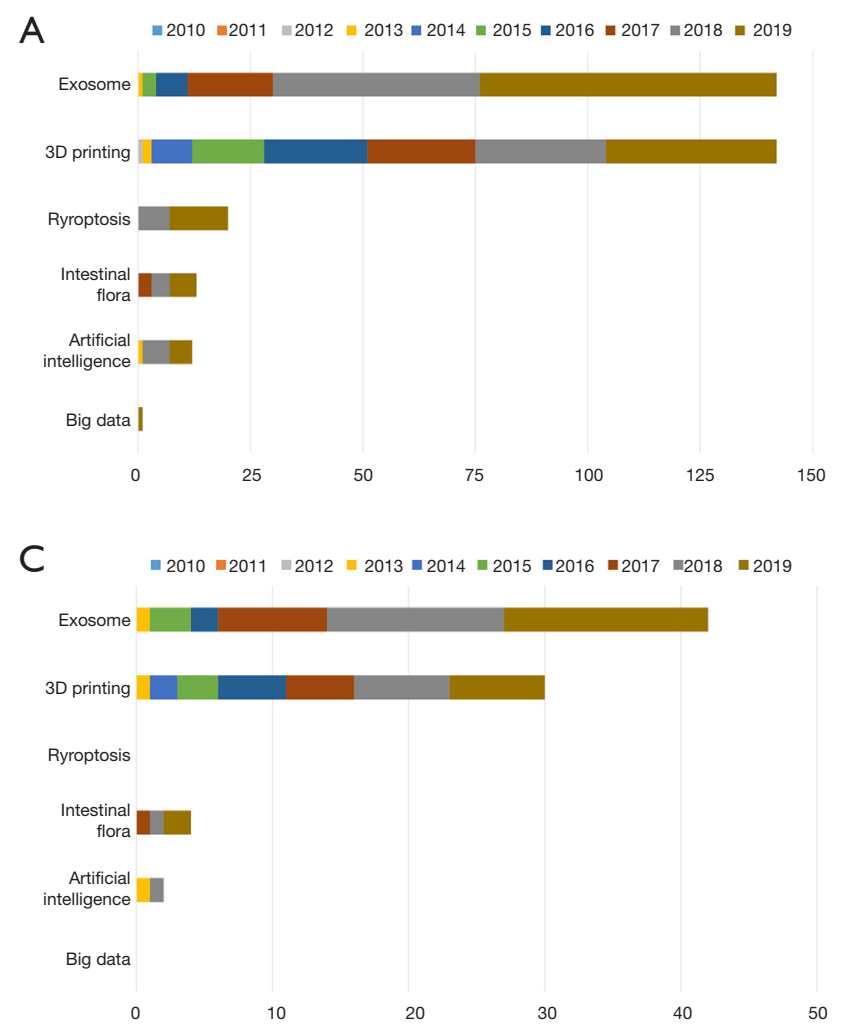

\section{B}

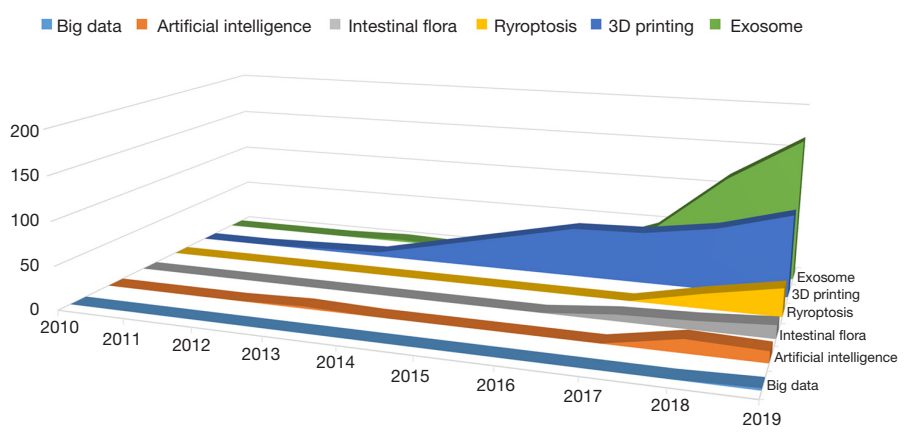

D

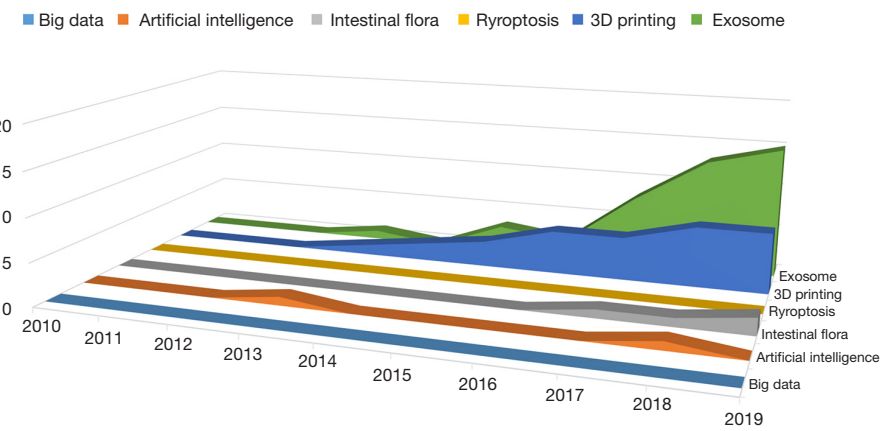

Figure 4 The keywords of emerging field in applications (A) and grants (C), and the number of projects involved in the keywords of emerging field in applications (B) and grants (D) for General Program in abnormalities and diseases of locomotor system over the past 10 years.

diseases. In the next few years, with the development of new methods and technologies, the research on the emerging scientific issues such as precise medicine, regenerative medicine and single-cell multi-omics in musculoskeletal fields are highly encouraged. The research based on clinical observation and translating basic discoveries into new drugs, treatments, and diagnostics are also encouraged. Moreover, the research focusing on the biological mechanisms of the interactions between musculoskeletal system and other systems or organs are preferentially encouraged.

\section{Conclusions}

In general, the numbers of applications and grants for General Program of NSFC in abnormalities and diseases of locomotor system have been increasing since the establishment of the Department of Health Sciences of NSFC. These projects, whether funded or not, were closely related to the key scientific questions and the cuttingedge discoveries of this field. Some emerging technologies, methods and theories were also well represented in these projects. In spite of that, the amount of applications and grants for General Program in this field are not large enough compared with other predominant medical disciplines, suggesting that the scale of scientific research in this field needs to be further strengthened. Because research work is too concentrated in a few hot areas, many key scientific issues have not received enough attention in this field. Additionally, the distribution of the funded projects among the host institutions is very uneven, indicating the imbalance of research teams and resources in this field. In order to overcome the above shortcomings, talents training and team building should be further strengthened in order to promote balanced development in this field, original research and application-driven basic research should be encouraged to enable breakthroughs, and transdisciplinary leading-edge research should be reinforced to facilitate the transformation.

\section{Acknowledgments}

Funding: None. 


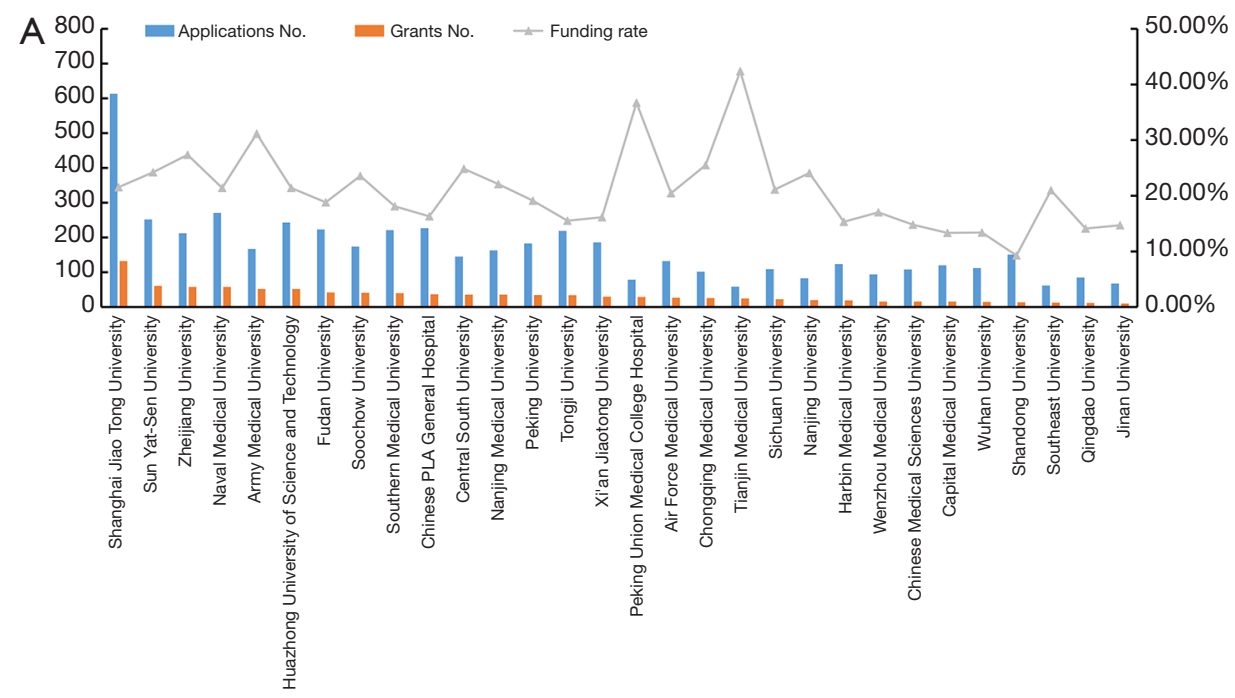

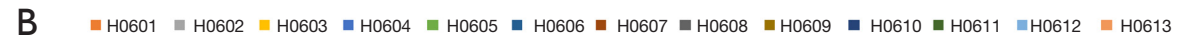

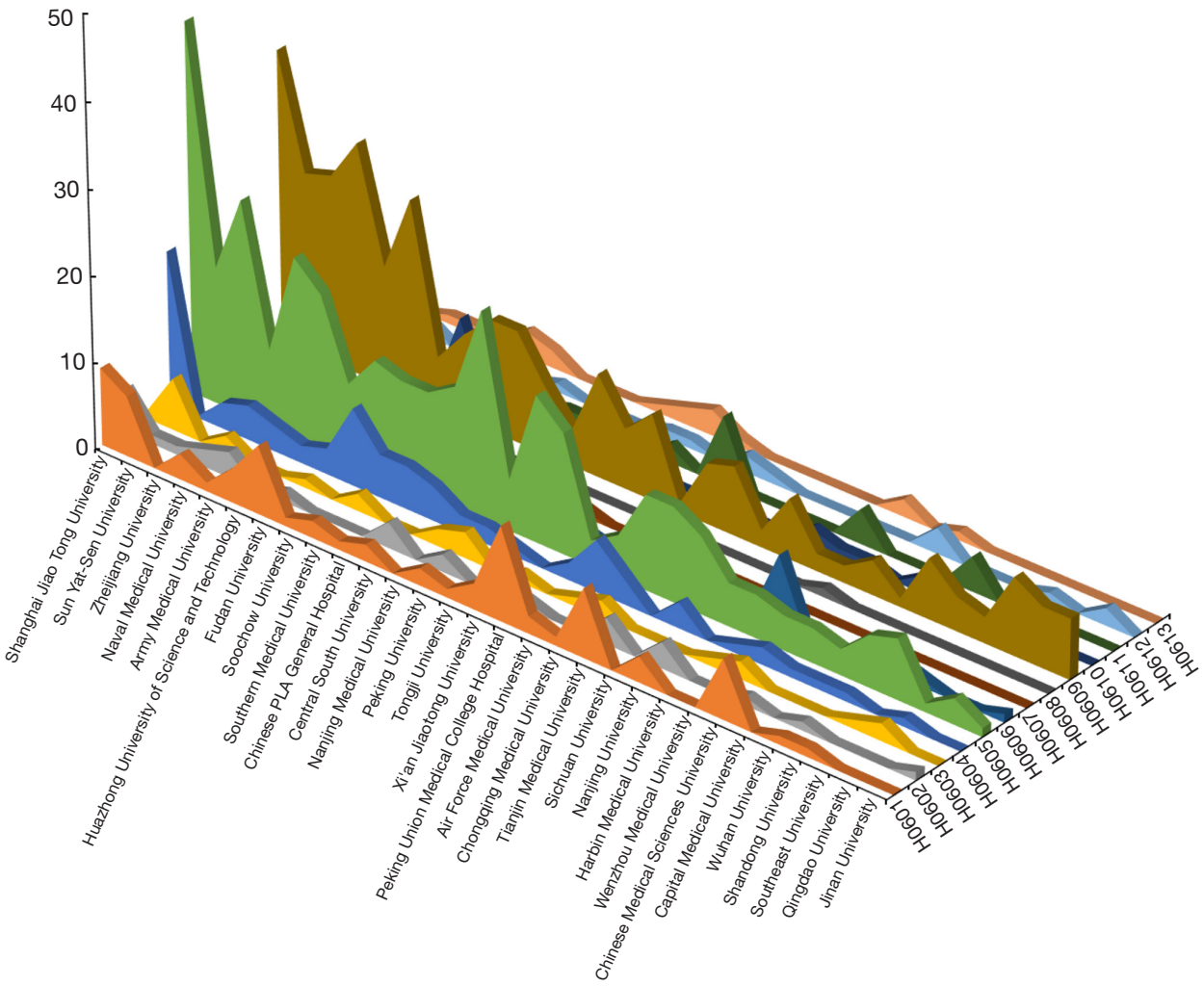

Figure 5 The number of applications, grants and funding rate of top 30 host institutions funded most for General Program in abnormalities and diseases of locomotor system over the past decade (A), and the distribution of the funded projects in the top 30 host institutions in the secondary application code of H06 (B). 


\section{Footnote}

Conflicts of Interest: The author has completed the ICMJE uniform disclosure form (available at http://dx.doi. org/10.21037/atm-20-3176). The author has no conflicts of interest to declare.

Ethical Statement: The author is accountable for all aspects of the work in ensuring that questions related to the accuracy or integrity of any part of the work are appropriately investigated and resolved.

Open Access Statement: This is an Open Access article distributed in accordance with the Creative Commons Attribution-NonCommercial-NoDerivs 4.0 International License (CC BY-NC-ND 4.0), which permits the noncommercial replication and distribution of the article with the strict proviso that no changes or edits are made and the original work is properly cited (including links to both the formal publication through the relevant DOI and the license). See: https://creativecommons.org/licenses/by-nc-nd/4.0/.

\section{References}

1. Geraghty J. Adenomatous polyposis coli and translational medicine. Lancet 1996;348:422.

Cite this article as: Dou D. Applications and grants of National Natural Scientific Foundation of China's General Program in abnormalities and diseases of locomotor system: a ten-year review. Ann Transl Med 2020;8(16):1024. doi: 10.21037/atm-20-3176
2. National Natural Science Foundation of China. National natural science fund guide to programs 2019. Beijing: Science Press, 2019.

3. Zhou M, Wang H, Zeng X, et al. Mortality, morbidity, and risk factors in China and its provinces, 1990-2017: a systematic analysis for the Global Burden of Disease Study 2017. Lancet 2019;394:1145-58.

4. Wu N, Ming X, Xiao J, et al. TBX6 null variants and a common hypomorphic allele in congenital scoliosis. $\mathrm{N}$ Engl J Med 2015;372:341-50.

5. National Natural Science Foundation of China. National natural science fund guide to programs 2020. Beijing: Science Press, 2020.

6. National Natural Science Foundation of China. National natural science fund guide to programs 2010. Beijing: Science Press, 2010.

7. National Natural Science Foundation of China. National natural science fund guide to programs 2013. Beijing: Science Press, 2013.

8. National Natural Science Foundation of China. National natural science fund guide to programs 2014. Beijing: Science Press, 2014.

9. Lin J, Chen L, Dou D. Progress of orthpaedic research in China over the last decade. J Orthop Translat 2020. doi: 10.1016/j.jot.2020.04.010. 